\title{
LA PROTECCIÓN DE LOS DERECHOS COLECTIVOS, EL PAPEL DE LA EMPRESA Y EL ESTADO, EN EL POSCONFLICTO COLOMBIANO: ENTRE LA REQUISITORIA DEL DERECHO INTERNACIONAL Y LAS VICISITUDES DEL DESARROLLISMO
}

\author{
Asier Tapias Gutiérrez ${ }^{1}$ \\ Berónica Narváez Mercado² \\ Jenny Alexandra Ocampo Castaño ${ }^{3}$
}

\section{Resumen}

En Colombia la responsabilidad internacional del Estado por la violación a los derechos colectivos ha avanzado considerablemente a partir de la Constitución Política de 1991, en ella se contempla una protección especial a los derechos colectivos que se ha desarrollado a través de la jurisprudencia de la Corte Constitucional principalmente respecto a el derecho al medio ambiente, sin embargo no siempre es el Estado es el que vulnera los derechos colectivos en algunos casos cuando los daños son causados por terceros sea empresas multinacionales o cualquier otra persona jurídica este adquiere

$1 \quad$ Magister en Derecho de la Universidad Pablo De Olavide. Magister en Ciencias Sociales de Universidad Del País Vasco. Magister en Estudios Internacionales de la Universidad del País Vasco. Docente Investigador de Universidad de Cartagena. Email: asiertg@hotmail.com

2 Doctorante en Derecho de la Universidad Libre de Colombia. MBA de la Escuela Libre de Derecho de Costa Rica, Abogada, Conciliadora y Docente Investigadora, Directora del Centro de Investigaciones Sociojurídicas de la Facultad de Derecho y Ciencias Políticas de la Corporación Universitaria del Caribe, Líder del Grupo de Investigación GISCER. Email: beronica.narvaez@cecar.edu.co

3 Estudiante de Derecho, 5 año, Universidad Libre de Pereira, integrante del Semillero de Derechos Humanos, correo: Alexa-19978@hotmail.com, celular: 3106518488. 
responsabilidad cuando se logre demostrar que existió complicidad, aquiescencia o falta de prevención por parte de los agentes estatales.

Palabras clave: responsabilidad social empresarial, derechos colectivos, derecho al medio ambiente, empresas.

\section{Abstract}

In Colombia, the international responsibility of the State for the violation of collective rights has advanced considerably since the Political Constitution of 1991, which provides for special protection of collective rights and jurisprudence mainly developed the right to the environment, without However, it is not always the State that violates collective rights in some cases when the damage is caused by third parties, whether multinational companies or any other legal entity acquires responsibility when it is demonstrated complicity, acquiescence or lack of prevention by the agents state

Key words: corporate social responsibility, collective rights, right to the environment, companies

\section{Introducción}

La responsabilidad del Estado ha ido aumentando progresivamente debido a que al principio el Estado era totalmente irresponsable ante los daños que le causaba a las personas y actualmente es posible establecer una responsabilidad estatal incluso de carácter internacional por las conductas que vulneren derechos humanos tanto por acción como por omisión, en consecuencia cuando el Estado o un tercero con la aquiescencia de este cause un daño se le impone la obligación de repararlo, es decir el derecho se ha desarrollado progresivamente para adaptarse a los nuevos cambios sociales, económicos y políticos de tal manera que el concepto de reparación integral implica una declaración de responsabilidad incluso de carácter internacional al Estado cuando incumpla alguna de sus obligaciones.

En virtud a lo anterior se analizará la responsabilidad del Estado en la protección de los derechos colectivos y como los terceros pueden también generar en algunos casos una responsabilidad de carácter estatal. 


\section{Responsabilidad del estado por hechos internacionalmente ilícitos resolución 53/86 de la asamblea general ONU}

En esta resolución se puede establecer las directrices para identificar cuando un hecho ilícito puede ser atribuible al Estado y para ello es necesario que en primer lugar que el Estado al momento de los hechos haya adquirido la obligación de carácter internacional y que el daño se produzca por acción u omisión de los agentes estatales, en consecuencia, el art 12 de esta resolución nos dice lo siguiente:

Artículo 12.- Existencia de violación de una obligación internacional. Hay violación de una obligación internacional por un Estado cuando un hecho de ese Estado no está en conformidad con lo que de él exige esa obligación, sea cual fuere el origen o la naturaleza de esa obligación. (1986)

Enlaresolución53de 1986 quesedefinecomohechosinternacionalmente ilícitos a la serie de acciones u omisiones que atentan contra los derechos humanosygeneran elincumplimiento de las obligacionesinternacionalesque haya adquirido el Estado, es decir estas acciones u omisiones sin importar o el origen o la naturaleza de la obligación en su conjunto conforman un hecho internacionalmente ilícito.

En este mismo orden de ideas a pesar de que esta resolución no se refiere expresamente a los casos en los cuales puede existir una responsabilidad estatal por las violaciones a los derechos humanos que generen terceros en su artículo 8 si hace referencia a las personas o grupo de personas que actúan bajo el control y la supervisión del Estado, es decir incluye tanto los casos en que terceros actúan con la aquiescencia del Estado y cuando por su omisión o falta de control se cometen graves violaciones a los derechos humanos por parte de estas personas, al respecto el articulo dice lo siguiente:

Artículo 8: Comportamiento bajo la dirección o control del Estado. Se considerará hecho del Estado según el derecho internacional el comportamiento de una persona o de un grupo de personas si esa persona o ese grupo de personas actúa de hecho por instrucciones o bajo la dirección o el control de ese Estado al observar ese comportamiento. (1986)

Es decir que si una persona o grupo de personas se encuentran la bajo la dirección y control del Estado y este no hace nada por evitar que sigan vulnerando los derechos humanos allí 
también se configura un incumplimiento a sus obligaciones internacionales lo que genera responsabilidad estatal, es por ello que este tema ha sido desarrollado en la jurisprudencia del Sistema Interamericano de Derechos Humanos principalmente en relación con las violaciones a los derechos humanos de allí la necesidad de analizar tres casos latinoamericanos para determinar las directrices que estableció la Corte Interamericana de Derechos Humanos (Corte IDH) para alegar una posible responsabilidad del Estado por hecho de terceros.

El primer caso es el de Masacre la Rochela Vs Colombia donde se declaró al Estado responsable internacionalmente por favorecer la impunidad de graves violaciones a los derechos humanos cometidas por los grupos paramilitares con el apoyo y la complicidad de los agentes estatales, al respecto la Corte IDH en la sentencia del 11 de mayo de 20007 se concluye lo siguiente:

La falta de una exhaustiva investigación sobre los mecanismos de operación de los paramilitares y sus vínculos y relaciones con agentes estatales, entre ellos miembros de la Fuerza Pública, ha sido uno de los factores que impidió la investigación, juicio y, en su caso, la sanción de todos los responsables. (2007)

En este caso podemos concluir que a pesar de que la masacre fue perpetuada por terceros reconocidos como grupos paramilitares se logró demostrar la responsabilidad internacional del Estado debido a que existía tolerancia e incluso complicidad por parte de agentes estatales que se evidencio en la falta de investigación sobre las operaciones paramilitares y los posibles vínculos que podían existir con miembros de la fuerza pública en la región, en consecuencia, se desviaron las investigaciones y no fue posible sancionar a todos los responsables.

El segundo caso es el caso González y otras ("Campo Algodonero") vs. México en la sentencia del 16 de noviembre de 2009 la Corte IDH hace referencia a la obligación que tiene el Estado de investigar los hechos cometidos por terceros, al respecto dice lo siguiente:

La Corte ha advertido que esta obligación se mantiene cualquiera sea el agente al cual pueda eventualmente atribuirse la violación, aún los particulares, pues, si sus hechos no son investigados con 
seriedad, resultarían, en cierto modo, auxiliados por el poder público, lo que comprometería la responsabilidad internacional del Estado. (2009)

Cabeconcluirqueenestecasose pudoevidenciarlafaltadeinvestigación que existía en los casos de homicidios de mujeres y la negligencia con la que actuaban los agentes estatales encargados de recibir las denuncias fue lo que ocasiono la responsabilidad del Estado Mexicano por la violación a sus obligaciones internacionales de protección a los derechos humanos de las tres jóvenes.

En este mismo orden de ideas el tercer caso Ximenes Lopes vs. Brasil es importante en la medida que hace referencia a la protección de los derechos de segunda generación, es decir el derecho a la salud y en su sentencia del 4 de julio contra el Estado de Brasil expresa lo siguiente:

La Corte considera que los Estados tienen el deber de regular y fiscalizar toda la asistencia de salud prestada a las personas bajo su jurisdicción, como deber especial de protección a la vida y a la integridad personal, independientemente de si la entidad que presta tales servicios es de carácter público o privado. (2006)

De acuerdo con los anteriores casos el Estado puede ser declarado internacionalmente responsable por los hechos de un tercero en primer lugar cuando se demuestre que el acto se cometió con tolerancia, complicidad o aquiescencia con personas o grupos de personas que causaron graves violaciones a los derechos y en segundo lugar cuando el Estado teniendo la obligación de investigar y prevenir el hecho de un particular no lo hizo a pesar de tener el deber de hacerlo.

Es por ello que cuando hablamos de terceros pueden ser gruposarmados al margen de la ley, empresas, organizaciones de personas o cualquier otro tipo de personas jurídicas de derecho privado es necesario analizar la relación que tiene el Estado con las personas jurídicas de derecho privado y como se les impone dentro del ámbito nacional e internacional la obligación a las empresas de proteger los derechos humanos. 


\section{El alcance dado a la relación Empresa - Estado en el derecho internacional.}

Es necesario entender que no sólo el Estado tiene la responsabilidad de prevenir las graves violaciones a los derechos humanos, sino que actualmente el derecho internacional ha reconocido la responsabilidad social que tienen las empresas en contribuir no sólo con el desarrollo sostenible a través de la implementación de tecnología limpias sino también en la protección de los derechos humanos de la sociedad.

UnejemploemblemáticoColombianodeloscasosenquemultinacionales han vulnerado los derechos humanos de los trabajadores y el Estado o ha sido cómplice de ello es la Masacre de las Bananeras ocurrida en Ciénaga, Magdalena, donde trabajadores de la United Fruit Company se negaron a continuar con su trabajo si la multinacional Estadunidense que aboliera los sistemas de contratación indirecta y les otorgara los beneficios que por ley tenían derecho, es así como después de un mes sin llegar a un acuerdo el 5 de diciembre de 1928 la huelga pacifica termino con el ataque indiscriminado de soldados colombianos que atacaron a sangre y fuego a los más de tres mil trabajadores que se encontraban reclamando mejores condiciones laborales esto desencadeno la ira del Congreso que fue quien termino denunciando que el Estado había acabado con la vida de miles de personas sólo por proteger los intereses de la Multinacional United Fruit Company.

En este mismo orden de ideas artículo de María Paula Alfonso Monroy, politólogacolombianase haceun recorridosobre cómose haido construyendo la responsabilidad de las empresas con los derechos humanos, al respecto nos dice:

Durante la década de 1980, las campañas de la sociedad civil denunciaron el perjudicial papel desempeñado por el Banco Mundial al apoyar grandes proyectos de desarrollo en muchos países de bajos ingresos, los que generaban destrucción medioambiental y menoscabo de derechos humanos. (Feeney, s.f., p. 11).

Es decir se empezó a desarrollar a partir de 1980 unas campañas por parte de la sociedad civil que tenía como objeto evidenciar las graves violaciones a los derechos humanos cometidas por las multinacionales pues estaban ocasionando en los países bajos daños al medio ambiente y los 
esquemas laborales atentaban contra los derechos de los trabajadores por lo que era necesario que los Estados adoptaran unas directrices que debían respetar las empresas multinacionales para que se generara un verdadero crecimiento económico y desarrollo social.

\section{El inicio de la responsabilidad social empresarial en el derecho internacional}

El primer instrumento internacional de responsabilidad social empresarial fue creado por la Organización de Cooperación y Desarrollo Económico (OCDE) que consisten en Líneas Directrices para Empresas Multinacionales que a pesar de no tener una fuerza vinculante, si son unas recomendaciones elaboradas por los gobiernos de 42 países que buscan compilar los principios y normas internacionales que deben implementar las multinacionales que tienen sede en sus territorios, además buscan promover una conducta de responsabilidad social respecto a la protección de los derechos humanos.

De acuerdo con OCDE es importante que las empresas multinacionales contribuyan con el desarrollo económico de un Estado, sin embargo, las actividades que estas desarrollen deben estar articuladas con las políticas públicas implementadas por el Estado en materia de cuidado, prevención y protección de los derechos humanos en su territorio, es por ello que en los principios generales de las Líneas Directrices para Empresas Multinacionales se le recomienda a las empresas adquirir los siguientes deberes:

Las empresas deberán: 1. Contribuir al progreso económico, social y medioambiental para lograr un desarrollo sostenible. 2. Respetar los derechos humanos internacionalmente reconocidos de las personas afectadas por sus actividades. (2011)

Cabe concluir que estas directrices a pesar de no tener una fuerza vinculante si es considerado en muchos Estados como un manual de conducta que deben seguir las empresas multinacionales que quieran desarrollar actividades en su territorio, además el concepto de la responsabilidad social empresarial ha ido desarrollándose a tal punto que hoy se habla de una obligación de la empresas el contribuir con el desarrollo sostenible y evitar que la actividad que desarrollan pueda afectar los derechos humanos de la sociedad. 
Es importante resaltar que dentro de estas Líneas Directrices para Empresas Multinacionales se creó un capitulo completo sobre Derechos Humanos en la medida que los Estados tienen la obligación de proteger los derechos humanos y para ello deben incorporar dentro de su ordenamiento jurídico principios que protejan a las personas de los abusos que puedan cometer las empresas multinacionales, al respecto en su capítulo IV dice lo siguiente:

Las empresas deberán: 1. Respetar los derechos humanos, lo cual significa que han de velar por no vulnerar los derechos de los demás y hacer frente a los impactos negativos sobre los derechos humanos en los que se vean implicadas. 2. En el marco de sus actividades propias, evitar causar impactos negativos sobre los derechos humanos o contribuir a que se generen y resolver dichos impactos si los hubiera. (2011)

Es decir, la obligación de las empresas con los derechos humanos es de hacer en la medida que en caso de existir en el desarrollo de sus actividades una vulneración a los derechos humanos de las personas debe reparar los daños que le causo a las personas y la otra obligación es la de abstenerse de causar impactos negativos al medio ambiente o a las personas siempre garantizando el progreso de la sociedad a través del desarrollo sostenible.

En este mismo orden de ideas la responsabilidad social empresarial no sólo se extiende a los derechos de primera y tercera generación, sino que también en estos principios internacionales se incluyen normas del Pacto Internacional de los Derechos Económicos, Sociales y Culturales principalmente en los derechos de los trabajadores pues en el 2000 el Consejo de Administración de la OIT adoptó la Declaración Tripartita de Principios sobre las empresas multinacionales y la política social con el cual se busca garantizar que exista un trabajo decente para todos, evitando la explotación y cualquier otro tipo de abusos por esquemas contractuales que vayan en contra de los derechos de los trabajadores reconocidos en el ámbito internacional.

En las políticas generales de la Declaración Tripartita de Principios sobre las empresas multinacionales y la política social en su numeral 10 inciso c encontramos la necesidad de que las empresas multinacionales 
se comprometan en el cumplimiento de los estándares internacionales de protección a los derechos de los trabajadores:

La responsabilidad de respetar los derechos humanos exige que las empresas, incluidas las empresas multinacionales dondequiera que operen: i) eviten que sus propias actividades provoquen o contribuyan a provocar consecuencias negativas y hagan frente a esas consecuencias cuando se produzcan, y ii) traten de prevenir o mitigar las consecuencias negativas sobre los derechos humanos directamente relacionadas con operaciones, productos o servicios prestados por sus relaciones comerciales, incluso cuando no hayan contribuido a generarlos. (2000)

Cabe resaltar que la responsabilidad social empresarial abarca también la protección de los derechos de los trabajadores de tal manera que se garanticen los derechos en el ámbito internacional, allí es necesaria la regulación del Estado para garantizar que estas directrices de derechos de los trabajadores en el ámbito internacional sea incorporada a su ordenamiento jurídico con el fin de prevenir posibles daños y abusos que puedan surgir en el desarrollo de las actividades de las empresas multinacionales.

En cuanto a la responsabilidad social empresaria en la protección de los derechos colectivos lo encontramos en los principios pacto mundial de las naciones unidas del año 2004 en el tema específicamente del medio ambiente creo tres principios que son necesarios que las empresas fomenten para la protección de los recursos naturales renovables y no renovables que pueden verse afectados con el desarrollo de sus actividades de explotación, estos principios son:

1. Las empresas deberán mantener un enfoque preventivo que favorezca el medio ambiente.2. Las empresas deben favorecer el desarrollo y la difusión de las tecnologías respetuosas con el medio ambiente. 3. Las empresas deben fomentar las iniciativas que promuevan una mayor responsabilidad ambiental. (2004)

Dado que la tecnología ha ido evolucionando y es posible hablar actualmente de tecnologías limpias y amigables con el medio ambiente se ha generado una responsabilidad a las empresas de implementar estas nuevas tecnologías, es decir las empresas deben procurar que sus actividades produzcan el menor daño posible al medio ambiente y a su vez proteger los 
derechos humanos de las personas, es decir en el caso de las comunidades indígenas es muy importante garantizar su derecho a la propiedad colectiva pues para ellossutierraes sagraday los Estados deben protegerestos territorios con mecanismos como la consulta previa o declarando los territorios como reservas naturales protegidas donde se prohíbe la explotación por cualquier tipo de empresa.

En el ámbito regional, aunque la Convención Americana de Derechos Humanos (CADH) no habla expresamente de los Derechos Colectivos jurisprudencialmente la Corte Interamericana de Derechos Humanos ha desarrollado el tema y ha creado un instrumento de protección de los derechos colectivos dirigido específicamente a las comunidades indígenas también conocido como la Declaración americana sobre los derechos de los pueblos indígenas que en su articulo XIX dice lo siguiente:

Artículo XIX. Derecho a la protección del medio ambiente sano 4. Los pueblos indígenas tienen derecho a la conservación y protección del medio ambiente y de la capacidad productiva de sus tierras o territorios y recursos. Los Estados deberán establecer y ejecutar programas de asistencia a los pueblos indígenas para asegurar esa conservación y protección, sin discriminación. (2016)

Es muy importante hacer este análisis porque los derechos colectivos en la jurisprudencia de la Corte IDH desempeña un papel complementario de los derechos humanos individuales y es obligación del Estado garantizar su pleno goce, en el caso de los territorios indígenas estos requieren de un enfoque étnico especial teniendo en cuenta las creencias y las necesidades de cada una de las etnias.

\section{Los avances de la responsabilidad social empresarial en Colombia}

En Colombia durante muchos años los Derechos Colectivos y del Medio ambiente se encuentran consagrados en el capítulo III de la Constitución Política y contempla no solo el el derecho a la paz, también el espacio público y en especial el derecho a un medio ambiente sano, al respecto dice lo siguiente: 
ARTICULO 79. Todas las personas tienen derecho a gozar de un ambiente sano. La ley garantizará la participación de la comunidad en las decisiones que puedan afectarlo. (1991)

Es decir, el derecho al medio ambiente goza de una protección constitucional y por lo tanto implican para el Estado la obligación de crear leyes e implementar mecanismos para su efectiva protección, sin embargo estos mecanismos no han evitado que terceros atenten contra el derecho a un ambiente sano de las personas, un ejemplo de ello son los atentados a los oleoductos perpetuados por el ELN, la minería ilegal de las Bandas Criminales y en algunos casos hasta las mismas empresas estatales producen estos daños como fue el caso de ECOPETROL quien causo el derrame de 22 barriles de petróleo sobre el rio Sogamoso en Barrancabermeja Santander lo que últimas termino contaminando el río y dos quebradas más con una mezcla crudo y lodo.

Uno de los avances más significativos que ha tenido el derecho al medio ambiente ha sido en la sentencia Sentencia T-622/16 donde la Corte Constitucional reconocio como sujeto de derechos al Río Atrato, esta sentencia es historica porque reconoce por primera vez al Rio Atrato como una entidad sujeta de derechos a la protección, conseracion y restauracion de Estado y las comunidades etnicas, además establece lo siguiente:

Esta interpretación encuentra plena justificación en el interés superior del medio ambiente que ha sido ampliamente desarrollado por la jurisprudencia constitucional y que está conformado por numerosas cláusulas constitucionales que constituyen lo que se ha denominado la "Constitución Ecológica" o "Constitución Verde". Este conjunto de disposiciones permite afirmar la trascendencia que tiene el medio ambiente sano y el vínculo de interdependencia con los seres humanos y el Estado (2016)

En conclusión, la Corte Constitucional reconoció que el hecho de ser sujetos de derechos va más allá del escenario humano y que la naturaleza en este caso el Rio Atrato tenía una protección especial constitucional que la llamaron la "Constitución Ecológica", en consecuencia con este reconocimiento el Estado tiene la obligación de protegerlo y recuperarlo pues como se dijo en la sentencia la transcendencia que tiene el medio ambiente 
en la salud, la vida y la integridad de una persona requiere que se tomen acciones contundentes en contra de los factores de contaminación que en este caso era la minería ilegal que se vive en el departamento del Chocó.

Finalmente, a pesar de los avances que han existido en materia jurisprudencia del derecho al medio ambiente y que en el ordenamiento jurídico existen mecanismos como la consulta previa para proteger los derechoscolectivosdelascomunidadesétnicasaún haymuypococompromiso social por parte de las empresas que desarrollan actividades de explotación de recursos no renovables de ahí la necesidad de aumentar los controles por parte del Ministerio del Medio Ambiente y promover en las empresas multinacionales el uso de tecnología limpias que no contaminen el medio ambiente.

\section{Conclusiones}

En conclusión, si una persona o grupo de personas se encuentran la bajo la dirección y control del Estado y este no hace nada por evitar que sigan vulnerando los derechos humanos allí también se configura un incumplimiento a sus obligaciones internacionales lo que genera responsabilidad estatal.

De acuerdo con los anteriores casos el Estado puede ser declarado internacionalmente responsable por los hechos de un tercero en primer lugar cuando se demuestre que el acto se cometió con tolerancia, complicidad o aquiescencia con personas o grupos de personas que causaron graves violaciones a los derechos y en segundo lugar cuando el Estado teniendo la obligación de investigar y prevenir el hecho de un particular no lo hizo a pesar de tener el deber de hacerlo.

Es necesario entender que no sólo el Estado tiene la responsabilidad de prevenir las graves violaciones a los derechos humanos, sino que actualmente el derecho internacional ha reconocido la responsabilidad social que tienen las empresas en contribuir no sólo con el desarrollo sostenible a través de la implementación de tecnología limpias sino también en la protección de los derechos humanos de la sociedad. 
De acuerdo con OCDE es importante que las empresas multinacionales contribuyan con el desarrollo económico de un Estado, sin embargo, las actividades que estas desarrollen deben estar articuladas con las políticas públicas implementadas por el Estado en materia de cuidado, prevención y protección de los derechos humanos en su territorio.

Cabe concluir que estas directrices a pesar de no tener una fuerza vinculante si es considerado en muchos Estados como un manual de conducta que deben seguir las empresas multinacionales que quieran desarrollar actividades en su territorio, además el concepto de la responsabilidad social empresarial ha ido desarrollándose a tal punto que hoy se habla de una obligación de la empresas el contribuir con el desarrollo sostenible y evitar que la actividad que desarrollan pueda afectar los derechos humanos de la sociedad.

La responsabilidad social empresarial abarca también la protección de los derechos de los trabajadores de tal manera que se garanticen los derechos en el ámbito internacional, allí es necesaria la regulación del Estado para garantizar que estas directrices de derechos de los trabajadores en el ámbito internacional sean incorporadas a su ordenamiento jurídico con el fin de prevenir posibles daños y abusos que puedan surgir en el desarrollo de las actividades de las empresas multinacionales.

Es muy importante hacer este análisis porque los derechos colectivos en la jurisprudencia de la Corte IDH desempeña un papel complementario de los derechos humanos individuales y es obligación del Estado garantizar su pleno goce, en el caso de los territorios indígenas estos requieren de un enfoque étnico especial teniendo en cuenta las creencias y las necesidades de cada una de las etnias.

El derecho al medio ambiente goza de una protección constitucional y por lo tanto implican para el Estado la obligación de crear leyes e implementar mecanismos para su efectiva protección.

La Corte Constitucional reconoció que el hecho de ser sujetos de derechos va más allá del escenario humano y que la naturaleza en este caso el Rio Atrato tenía una protección especial constitucional que la llamaron la "Constitución Ecológica", en consecuencia, con este reconocimiento el Estado tiene la obligación de protegerlo y recuperarlo pues como se dijo en 
la sentencia la transcendencia que tiene el medio ambiente en la salud, la vida y la integridad de las personas.

A pesar de los avances que han existido en materia jurisprudencia del derecho al medio ambiente y que en el ordenamiento jurídico existen mecanismos como la consulta previa para proteger los derechos colectivos de las comunidades étnicas aún hay muy poco compromiso social por parte de las empresas que desarrollan actividades de explotación de recursos no renovables de ahí la necesidad de aumentar los controles por parte del Ministerio del Medio Ambiente y promover en las empresas multinacionales el uso de tecnología limpias que no contaminen el medio ambiente.

\section{Referencias}

ONU. Asamblea de las Naciones Unidas. Resolución 53/86. RESPONSABILIDAD DEL ESTADO POR HECHOS INTERNACIONALMENTE ILÍCITOS. (1986). Art 12. Disponible en: https://www. dipublico.org/4076/responsabilidad-del-estado-por-hechos-internacionalmente-ilicitos-ag5683/.

Corte IDH. Caso de la Masacre de La Rochela Vs. Colombia. Fondo, Reparaciones y Costas. Sentencia de 11 de mayo de 2007. Serie C No. 163. Párr.164. Disponible en: http://www.corteidh.or.cr/docs/casos/articulos/seriec_163_esp.pdf.

Corte IDH. Caso González y otras ("Campo Algodonero") Vs. México. Excepción Preliminar, Fondo, Reparaciones y Costas. Sentencia de 16 de noviembre de 2009. Serie C No. 205.Párr 291. Disponible en: http://www.corteidh.or.cr/docs/casos/articulos/seriec_205_esp. pdf.

Corte IDH. Caso Ximenes Lopes Vs. Brasil. Sentencia de 4 de julio de 2006. Serie C No. 149. Párr. 89. Disponible en: http://www.corteidh.or. cr/docs/casos/articulos/Seriec_149_esp.pdf.

Colombia Informa. (2017). la masacre de las bananeras: la matanza que, si ocurrió, 16 de junio de 2017, pag 1. Bogotá. Disponible en: http:// www.colombiainforma.info/5-y-6-de-diciembre-la-masacre-delas-bananeras-la-matanza-que-si-ocurrio/

Alfonso MP, (2013). Empresas y Derechos Humanos: una responsabilidad en construcción. RevistaFACULTADDEDERECHOYCIENCIASPO- 
LÍTICAS. Vol. 43, No. 118 / pág. 6. Medellín. Disponible en: http:// www.scielo.org.co/pdf/rfdcp/v43n118/v43n118al1.pdf.

OCDE (2013). Líneas Directrices de la OCDE para Empresas Multinacionales, OECD Publishing. Pag 22 - 35 Disponible en: http://www. oecd.org/daf/inv/mne/MNEguidelinesESPANOL.pdf.

OIT (2017), Declaración Tripartita de Principios sobre las Empresas Multinacionales y la Política Social. Pag 13. Disponible en: https:// www.ilo.org/wcmsp5/groups/public/---ed_emp/---emp_ent/documents/publication/wcms_124924.pdf.

OEA.(2016).DECLARACIÓNAMERICANASOBRELOSDERECHOSDELOS PUEBLOS INDÍGENAS. AG/RES. 2888 (XLVI-O/16).Pag 8. Disponible en: http://www.oas.org/es/sadye/documentos/res-2888-16es.pdf.

EL ESPECTADOR. (2018). Anla abre investigación contra Ecopetrol por derrame de petróleo en Barrancabermeja. (Santander). Pag 1. Disponible en : https://www.elespectador.com/noticias/medio-ambiente/anla-abre-investigacion-contra-ecopetrol-por-derrame-de-petroleo-en-barrancabermeja-articulo-745785.

CORTE CONSTITUCIONAL. (2016).MP: JORGE IVÁN PALACIO PALACIO. Sentencia T-622/16. Disponible en: http://www.corteconstitucional.gov.co/relatoria/2016/t-622-16.htm 